\title{
Maintenance of neutralizing SARS-CoV-2 antibodies over five months in convalescent SARS-CoV-2 afflicted patients.
}

\author{
Sissy Sonnleitner ${ }^{1}$, Martina Prelog ${ }^{2}$, Bainca Jansen ${ }^{1}$, Chantal Rodgarkia-Dara ${ }^{3}$, Sarah \\ Gietl $^{1}$, Carmen Schönegger ${ }^{1}$, Stephan Koblmüller ${ }^{4}$, Christian Sturmbauer ${ }^{4}$, Wilfried \\ Posch $^{5}$, Giovanni Almanzar ${ }^{2}$, Hanna Jury ${ }^{2}$, Norbert Nowotny ${ }^{6}$, and Gernot Walder ${ }^{1}$ \\ ${ }^{1}$ Dr. Gernot Walder GmbH \\ ${ }^{2}$ University Hospital Wurzburg \\ ${ }^{3}$ THP Medical products \\ ${ }^{4}$ University of Graz \\ ${ }^{5}$ Medical University of Innsbruck \\ ${ }^{6}$ Mohammed Bin Rashid University of Medicine and Health Sciences
}

December 11, 2020

\begin{abstract}
Level and duration of protective immunity against SARS-CoV-2 after primary infection is of crucial importance for preventive approaches. In order to provide evidence for the longevity of specific antibodies, we investigated the generation and maintenance of neutralizing antibodies of convalescent SARS-CoV-2-afflicted patients over a five month period post primary infection using an immunofluorescence assay, a commercial chemiluminescent immunoassay and an in-house enzyme-linked plaque-reduction neutralization assay. We present the successful application of an improved version of the plaque-reduction neutralization assay, which can be analyzed optometrically, significantly simplifying the interpretation of the results. Based on the results of the plaque-reduction neutralization assay, neutralizing antibodies were maintained in $85.3 \%$ of convalescent individuals without significant decay over five months. Furthermore, a positive correlation between severity of infection and neutralizing titer was shown. In conclusion, SARS-CoV-2-afflicted individuals have been proven to be able to establish and maintain neutralizing antibodies over a five months' period after primary infection which allows to hope for long-lasting presumably protective humoral immunity after wild-type infection or even after vaccination.
\end{abstract}

Maintenance of neutralizing SARS-CoV-2 antibodies over five months in convalescent SARSCoV-2 afflicted patients.

Short title: Serologic study on the longevity of humoral immunity against SARS-CoV-2.

Sissy Therese Sonnleitner ${ }^{1,3}$, Martina Prelog ${ }^{4}$, Bianca Jansen ${ }^{1}$, Chantal Rodgarkia-Dara ${ }^{5}$, Sarah Gietl ${ }^{1}$, Carmen Maria Schönegger ${ }^{1}$, Stephan Koblmüller ${ }^{2}$, Christian Sturmbauer ${ }^{2}$, Wilfried Posch $^{3}$, Giovanni Almanzar ${ }^{4}$, Hanna Jury ${ }^{4}$, Norbert Nowotny ${ }^{6,7}$, Gernot Walder ${ }^{1}$.

${ }^{1}$ Dr. Gernot Walder GmbH, Medical Laboratory, Department of Virology, 9931 Ausservillgraten 30, Austria. sissy.lamprecht@infektiologie.tirol

${ }^{2}$ Institute of Biology, University of Graz, Universitaetsplatz 2, 8010 Graz, Austria.

${ }^{3}$ Division of Hygiene and Medical Microbiology, Medical University of Innsbruck, 6020 Innsbruck, Austria.

${ }^{4}$ University Hospital Wuerzburg, Department of Pediatrics, University of Wuerzburg, Josef-Schneider-Str. 2, Wuerzburg, Germany. 
5 THP Medical products, Shuttleworthstrasse 19, 1210 Vienna, Austria.

${ }^{6}$ Viral Zoonoses, Emerging and Vector-Borne Infections Group, Institute of Virology, University of Veterinary Medicine Vienna, Veterinaerplatz 1, 1210 Vienna, Austria.

${ }^{7}$ Department of Basic Medical Sciences, College of Medicine, Mohammed Bin Rashid University of Medicine and Health Sciences, Building 14, Dubai Healthcare City, Dubai, United Arab Emirates.

\section{ABSTRACT}

Level and duration of protective immunity against SARS-CoV-2 after primary infection is of crucial importance for preventive approaches. In order to provide evidence for the longevity of specific antibodies, we investigated the generation and maintenance of neutralizing antibodies of convalescent SARS-CoV-2-afflicted patients over a five month period post primary infection using an immunofluorescence assay, a commercial chemiluminescent immunoassay and an in-house enzyme-linked plaque-reduction neutralization assay.

We present the successful application of an improved version of the plaque-reduction neutralization assay, which can be analyzed optometrically, significantly simplifying the interpretation of the results. Based on the results of the plaque-reduction neutralization assay, neutralizing antibodies were maintained in $85.3 \%$ of convalescent individuals without significant decay over five months. Furthermore, a positive correlation between severity of infection and neutralizing titer was shown.

In conclusion, SARS-CoV-2-afflicted individuals have been proven to be able to establish and maintain neutralizing antibodies over a five months' period after primary infection which allows to hope for longlasting presumably protective humoral immunity after wild-type infection or even after vaccination.

Keywords: SARS-CoV-2, immunity, longevity, neutralization test, IgG antibodies

\section{INTRODUCTION}

Little is known about persistence of the immunologic footprint left in a convalescent SARS-CoV-2 afflicted patient and first publications showed discrepancies concerning the longevity of specific IgG antibodies . The development of specific antibodies in the acute phase of COVID-19 is well documented and most patients display specific antibody responses between day 10 and day 21 post infection (Kellam \& Barclay, 2020; Long et al., 2020). Whereas milder courses of disease may result in a delayed generation of antibodies, in a small number of cases, patients may even stay antibody-negative after infection with SARS-CoV-2 . In comparable studies, seropositivity rate reached up to $90 \%$ and $100 \%$ Studies investigating the persistence of the antibody response are rare, but it is known that antibodies to other coronaviruses wane over time, in the range of $12-$ 52 weeks from the onset of symptoms onwards (. Short follow-up studies showed preservation of SARS-CoV-2 IgG antibody levels over the course of seven weeks (Xiao et al., 2020), at least in $80 \%$ of patients (Jianyun et al., 2020). In comparison, $90 \%$ and $50 \%$ of SARS-CoV-1 infected patients have been shown to maintain $\operatorname{IgG}$ antibodies for two and three years, respectively (.

Short-term immunity is defined as the wane of specific antibodies after a period of about 40 weeks, which leads to annual or otherwise periodic outbreaks as is well-known from nonSARS-like human coronaviruses (nonSARS-like hCoVs) as well as influenza viruses. The knowledge of duration of protective immunity against SARS-CoV-2 after primary infection is of great importance for the understanding of possible future scenarios of herd protection after wild-type infection or vaccination.

In this study, we accompanied 34 volunteers representing the first SARS-CoV-2 afflicted patients in East Tyrol and investigated the course of specific antibody responses beginning in convalescence 21-43 days after the onset of symptoms until 5 months (22 weeks) later.

In order to analyze potentially protective neutralizing IgG antibodies against SARS-CoV-2, we developed and introduced a fast and reliable novel enzyme-linked neutralization assay (ELNA) that allows the determination of neutralization antibodies within 30 hours.

MATERIAL \& METHODS 


\section{Enrolment and sample collection}

Thirty-four volunteers in East Tyrol with PCR-confirmed SARS-CoV-2 infections and mostly clinically manifested COVID-19 symptoms (31/34) were accompanied over 22 weeks post infection after informed consent was taken from the volunteers. Ethical approval of the study was obtained by the Ethics Committee of the University Hospital Wuerzburg no. 20201105_01.

Following the time range of initial data collection, we conducted serological follow-up examinations to evaluate possible recurrences and seroconversion rates. First blood draw occurred 21 - 43 days (median 35 days, standard deviation 15.6 days) after the onset of symptoms in the first week of April 2020, followed by further assessments during the investigative period, in the following called T1 - T4, and a final blood draw 5 and 6 months post infection in August and September 2020, respectively (T5). Clinical data were obtained using a standardised data collection form.

The presence of IgG-anti-spike1/spike2 and neutralizing antibodies was analyzed in follow-up serum samples by four different serologic methods to ensure validity of results: IgG in-house immunofluorescence assay (IFA), IgM in-house IFA, CLIA IGG®) SARS-CoV-2 S1/S2 IgG (DiaSorin S.p.A., Saluggia, Italy) (CLIA IGG; clinical sensitivity 97.1\%; 95\% CI: 85.5 - 99.5\%, clinical specificity 100\%; 95\% CI: $96.5-100 \%$ as reported by the manufacturer) and enzyme-linked neutralization assay (ELNA).

\section{$R N A$ extraction and $R T-q P C R$ :}

Sample drawing was performed by trained healthcare personnel via nasopharyngeal swab. Immediately after collection, viral RNA was extracted using the Indimag Pathogen kit (Indical Bioscience GmbH, Germany) and tested for SARS-CoV-2 by RT-qPCR using the Bio-Rad CFX96 system (Roche, Switzerland) with a LightMix Modular Assay kit in accordance with the modified Charité guidelines . $10 \mu$ l of extracted RNA were added into $15 \mu \mathrm{l}$ reaction mixture (mastermix). Each $15 \mu \mathrm{l}$ mastermix contained $12.5 \mu \mathrm{l}$ buffer solution, $0.25 \mu \mathrm{l}$ enzyme mix, $1.75 \mu$ l of nuclease-free water and $0.5 \mu$ l primer probe wHCoV (ORF1ab: E-Gene, occasionally $\mathrm{N}$-Gene/Rd-Gene). Reactions were incubated at $55^{\circ} \mathrm{C}$ for 5 minutes and $95^{\circ} \mathrm{C}$ for 5 minutes in order to conduct reverse transcription of viral RNA, sample denaturation and enzyme activation. These steps were followed by PCR-amplification with 45 cycles at $95^{\circ} \mathrm{C}$ for $5 \mathrm{~s}, 60^{\circ} \mathrm{C}$ for $15 \mathrm{~s}$ and $72^{\circ} \mathrm{C}$ for $15 \mathrm{~s}$. Cooling was implemented at $10^{\circ} \mathrm{C}$ for $30 \mathrm{~s}$.

Results were interpreted based on the Second derivative maximum (SDM) method (Tichopad et al., 2003). Positive results were confirmed by RdRp- and N-gene (Corman et al., 2020) RT-qPCR. Samples with an initial $\mathrm{Cq}$ value lower than or equal to 37 were assigned to repeated testing including extraction. A Cq value higher than 40 was defined negative.

\section{Isolation of SARS-CoV-2:}

Isolation of SARS-CoV-2 was performed from nasopharyngeal swabs after obtaining positive RT-qPCR results by inoculation on VeroB4 (no. ACC-33, DSMZ) in T25 tissue culture flasks (Sarstedt, Germany) for $1 \mathrm{~h}$ at $35^{\circ} \mathrm{C}$. After incubation, the sample was removed and Medium199 (Gibco, USA) with $2.5 \%$ fetal calf serum (FCS; Gibco, USA) and a mixture of antibiotics (streptomycin, vancomycin, penicillin, each $1 \mu \mathrm{g} / \mathrm{ml}$ ) was added. We monitored virus cultures daily for cytopathic effects and tested for specific viral RNA every three days. Isolation was considered successful when cytopathic effect was 80 to $100 \%$ in passage 0 as well as passage 1 and/or $\mathrm{Cq}$ value in qPCR was lower than 20. Highly positive supernatants were harvested, centrifuged at 3,400g for 5 minutes and stored at $-80^{\circ} \mathrm{C}$ in $10 \% \mathrm{FCS}$. A further passage of diverse isolates was performed to obtain the highest possible concentration, which was Cq 14 on average. All work involving infectious SARS-CoV-2 was carried out in a BSL3 facility, following the institutional guidelines and regulations. Whole genome sequencing was carried out by Eurofins Genomics, Germany, and a frequent local genotype (no. 6893) was used for neutralization assay.

In-house immunofluorescence assays (IFA) were assembled as described elsewhere . In brief, VeroB4 cells were infected with local SARS-CoV-2 strain 6893 and fixed on IFA slides after three days using ice-cold acetone-methanol (1:1). 
The LIAISON@ SARS-CoV-2 S1/S2 IgG (DiaSorin S.p.A., Saluggia, Italy) (LIAISON) is a CLIA (Chemiluminescent Immunoassay) which detects IgG antibodies reactive with the spike protein (S1/S2 domain).

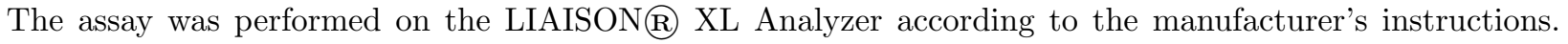
The diagnostic sensitivity was $97.9 \%(89.1 \%$ - 99.6\%; Wilson $95 \% \mathrm{Cl})$ according to the manufacturer, the specificity in laboratory routine was $99.0 \%(96.4 \%$ - 99.7\%; Wilson $95 \% \mathrm{Cl})$.

Enzyme-Linked Neutralization Assay (ELNA):

VeroB4 cells (ACC-33, DSMZ) were seeded in flat-bottom 96 well plates (Sarstedt, Germany) with Medium199 (Thermo Scientific Gibco, USA) and 10\% fetal calf serum (Thermo Scientific Gibco, USA) at a density of about $10^{6}$ cells $/ \mathrm{ml}$ to receive a confluent monolayer. Next day, an infectivity titration was carried out to determine 100 tissue culture infectious dose $50 \%$ (100 TCID $\left._{50}\right)$.

Inactivated serum samples were titrated in duplicate in twofold dilution steps, starting at a dilution of 1:4 in Medium199 containing 3\% fetal calf serum. Equal volumes of virus $\left(1 \times 10^{5} \mathrm{TCID}_{50}\right)$ and serum dilutions in Medium199 were mixed and subsequently incubated for 1 to 1.5 hours at room temperature in U-bottom 96 well plates (Thermo Scientific Thermo Scientific Nunc, USA). After incubation, a pre-seeded flat-bottom 96 well plate with confluent VeroB4 cells was used, medium was discarded, the incubated mixture of patient's serum and defined virus solution was transferred to each corresponding well of the flat-bottom plate and the plate was incubated for 24 hours at room temperature. Incubation was stopped by discarding supernatant, cells were washed in PBS twice, fixed with ice-cold acetone-methanol (1:1) and frozen for at least 15 minutes. All steps were performed under strict observation and in compliance with biosafety level 3 . The analysis was carried out like an enzyme-linked immunosorbent assay using a BEP III (Siemens, Germany) according to the following steps: blocking (45 minutes, $37^{\circ} \mathrm{C}$, Candor Biosciences, Germany), washing $3 \mathrm{x}$ (wash pod, Siemens, Germany), anti-SARS-CoV-2 nucleocapsid protein IgG (Bioss bsm-41413M, dilution 1:5000) for 30 min. at $37^{\circ} \mathrm{C}$ ), washing $3 \mathrm{x}$, adding of horseradish-peroxidase-conjugated goat anti mouse IgG (ABIN376241, dilution 1:5000) for $30 \mathrm{~min}$. at $37^{\circ} \mathrm{C}$ ), washing $3 \mathrm{x}$, adding substrate tetramethylbenzidine (TMB) and stop solution (Siemens, Germany). 24 hours post infection the reading of the results was done.

The cut-off titer was set by titrating defined negative human sera from volunteers out of healthy Tyrolean blood donors from the year 2009 and was set at 1:4 in combination with the viral dose of $1 \times 10^{5}$ TCID $_{50}$ and calculated as median optic density minus the standard deviation. A sample was considered positive when the given optic density was higher than the cut-off titer.

Titers of single 1:4 in ELNA were valued as borderline, titers of 1:4 in duplicate as weak neutralization ability, 1:8 to $1: 16$ as moderate/good and $>1: 16$ as strong neutralizing ability.

Definition of antibody development:

In ELNA and IFA, a change in antibody titer of more than one dilution step over the investigation period from time point 1 (T1) to time point 5 (T5) was defined as an increase or decrease of the titer. In the IgG CLIA assay, the development of antibodies was defined as a change in chemoluminescence of more than $50 \%$.

Statistics:

Dichotomous data were tested by a chi-squared test. A two-sided significance level of $p=0.05$ was used for determining statistical significance. The Pearson correlation coefficient was used to analyze correlation of titers between CLIA and ELNA (Microsoft@ Excel@, Microsoft 395 MSO, Windows 2010). To determine

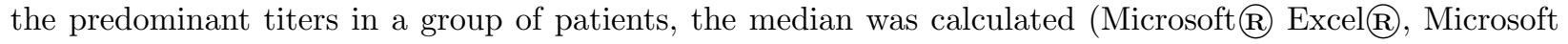
395 MSO, Windows 2010).

\section{RESULTS}

Characteristics of the study group:

The individual severity of disease was assessed clinically using a standardized questionnaire including age, gender, pre-existing as well as acute physical condition and rated as asymptomatic, mild, moderate and 
severe course of disease according to the definitions by Gietl et al., under review).

Within our study group, three patients (8.8\%) were asymptomatically infected with SARS-CoV-2, 20 patients $(58.8 \%)$ showed a mild, six (18.8\%) a moderate and five (14.7\%) a severe course of the disease.

In total, the group consisted of 18 women and 16 men. Gender-related differences in the course of disease was not significant $(p=0.11)$, although only one $(5.6 \%)$ female had a severe course compared to four male patients $(25 \%)$.

The median age was 49.0 years (range 11 to 77 years; $s d=16.4$ ). Due to different expectations of disease course (Gietl et al., under review), the age groups were divided into three groups of $11-30,31-60$ and 61 -77 years.

Seroconversion assessed by different methods:

90.9\% (30 of 33) SARS-CoV-2-positive patients in the convalescent phase ( $>21 \mathrm{~d}$ after onset of the symptoms) tested had seroconverted at T1 in April and in total, $85.3 \%$ of the patients (29 of 34 samples) had seroconverted until September, as determined by neutralization test (table 1).

Overall, $85.3 \%$ of SARS-CoV-2-infected persons seroconverted and maintained constant neutralizing antibodies over the study period of five months.

The highest number of positives was found in the IgG IFA one month post infection (32 of 34; 94.1\%), the lowest number of seroconversions was found in CLIA IgG (28 of 34; 82.4\%). In 11 cases (32.4\%), IgM was detectable by IFA $28-41$ days after onset of the symptoms.

Four patients had no specific antibody response at all and eleven patients developed only weak antibody responses five months post infection. All four negative patients had a mild course of disease; their mean age was $51(s d=12.4)$ years.

Comparison CLIA IgG-ELNA

Both methods showed that specific antibodies stayed constant over the observation period and that a stronger disease severity led to a more stable antibody response.

CLIA IgG recognized two sera from the first time point as positive $(5.9 \%)$, which turned out as negative in ELNA. This result goes along with the manufacturer's information concerning 95\% specificity and 0\% false-negative results. Repeated serologic investigations at T5 exhibited one patient (3.0\%) false-negative in CLIA IgG.

Comparison IFA - ELNA

IFA gave 94\% positive results, 6 more than ELNA, in the sera of T1 and the highest decrease of antibodies until T5 $(\mathrm{n}=7 ; 65.6 \%$; decrease of $28.4 \%)$. Two of the seven sera, which proved negative in IFA, were also negative in ELNA, the others were weak positive with titers around 1:4 in ELNA, as shown in table 2.

Longevity of IgG antibodies:

In one 65 year old male patient with a mild course of disease the neutralizing antibody titer dropped from $1: 32$ at $\mathrm{T} 1$ to $<1: 8$ at $\mathrm{T} 5$.

Two patients showed an increase in neutralizing antibodies over the sample period. In both patients, the first blood sample showed weak or moderate neutralizing ability with titers of 1:4 and 1:8, which increased to titers of 1:32 and 1:64, respectively. The courses of disease in these cases were mild and severe, respectively.

In $83.9 \%$ of the patients, the neutralizing antibody titers against SARS-CoV-2 stayed constant and did not change more than two dilution steps or more than $50 \%$ change of optic density in CLIA IgG.

Two patients were negative in the CLIA IgG assay but showed weak neutralizing activities with antibody titers of $1: 4$. 


\section{Influence of the course of disease on neutralizing antibodies:}

The median neutralizing antibody titer of symptomatic patients was two levels higher than in asymptomatic patients $(\mathrm{n}=31$ versus 3 patients;Figure $\mathbf{1})$. However, there was no statistically significant correlation between the individual titers and the severity of infection $(k=-0.09)$.

Our data also reveal a relation between the severity of infection and neutralizing activity by ELNA (3 and $31 / 34 ; p=0.07)$.

Moderate or severe SARS-CoV-2 infections led to the development of neutralizing antibodies significantly more frequently than in asymptomatic or mild infections $(p=0.03)$. There was no significant difference in neutralization activities between asymptomatic and mild courses of disease ( $p=0.17)$. Interestingly, $60 \%$ of the patients who suffered severe infections (3/5), developed low neutralizing antibodies (Figure 2 ).

Age and disease severity:

There was no correlation between age and disease severity $(k=0.09)$ or neutralizing antibodies and disease severity $(k=-0,0951)$, nor could we find significant predominance of severe cases in older age groups or male patients $(p=0.53)$.

The mean age of severe cases was $46(s d 15.1, \mathrm{n}=5)$ versus 49 years (sd $16.8 ; p=0.36$, not significant) in cases of other severity categories. All patients with severe infections had neutralizing antibodies at T1. However, only $2(40 \%)$ developed constantly neutralizing antibodies, and $60 \%$ in this group showed low titers $(<1: 8) 5$ months post infection.

\section{DISCUSSION}

Specific neutralizing antibodies were detected in $90.0 \%$ of 34 laboratory confirmed cases of SARS-CoV-2 infected patients in the early convalescent phase 21 days post infection and stayed constant in $85.3 \%$ of the patients in the convalescent stage five months later, confirming recently published data of high prevalence of neutralizing antibodies in most SARS-CoV-2 afflicted individuals

In our study, neutralization activity was correlated with disease severity. $86.7 \%$ of the symptomatic patients (21 of 31) as well as $66.7 \%$ of the asymptomatic patients (1 of 3) maintained neutralizing antibodies over the observation period of 5 months. Investigations of IgG levels and neutralizing antibodies in early phase of convalescence gave a similar result (. $60 \%$ of the study members who suffered severe infections $(3 / 5)$ developed low neutralizing antibody titers, which may allow the speculation that impaired immune responses may contribute to severe disease manifestation or an unevently observed B cell lymphopenia in COVID-19 (Melenotte et al., 2020; Ni et al., 2020; Q. X. Long et al., 2020; Wu et al., 2020; Qin et al., 2020). Although, concomitant treatments e. g. with glucocorticoids or other immunosuppressants recommended for COVID-19 may mitigate the generation of an efficient humoral immune response (Bamoulid et al., 2015; Thaunat et al., 2016).

In accordance with another study, the IgG levels in the symptomatic group $(\mathrm{n}=31)$ were significantly higher than those in the asymptomatic group $(\mathrm{n}=3 ; p=0.008)$.

Two patients were negative in the early stage of the convalescent phase four weeks post infection (T1), one - a patient with a mild course of disease - stayed negative in all tests, whereas another one with an asymptomatic infection developed a weak neutralization response in the late convalescent phase (T5). This also fits well with published data, where milder courses of disease may cause longer periods to generate specific antibodies and in a low number of cases, patients did not seroconvert at all after infection with SARS-CoV-2

A recently published study (Wajnberg et al., 2020) investigated the persistence of neutralizing antibodies and is in accordance with our results of relatively stable maintenance of neutralizing antibodies in convalescent COVID-19 patients over a study period of 5 months.

The comparison of different serologic methods shows that the IgG CLIA reflects the results of the neutralization assay well with concordances of $95 \%$, giving overall $5.9 \%$ false-positive and $3 \%$ false-negative results. 
The discrepancies between the two methods may be found in the coating with Spike S1 and S2, but the Receptor Binding Domaine (RBD), which is expressed in the NT results, is of great significance, too. It is also discussed by others (Suthar et al., 2020).

Many commercially available SARS-CoV-2 ELISAs are coated with Spike 1, 2 or both, but neutralizing antibodies are directed against the RBD or other targets and more unspecific antibodies tend to decline in the convalescent phase of an infection (Suthar et al., 2020). A high ELNA titer does not necessarily mean a high CLIA titer and vice versa.

The CLIA IgG is a fast and easy to handle diagnostic tool, which is known to have limited specificity. The high number of positives at the first time point might be due to the low specificity of the method and suggests a high degree auf cross-reactivity, presumably with endemic nonSARS-hCoVs like OC43.

Our results show that a high proportion of convalescent SARS-CoV-2 afflicted patients maintain constant neutralizing antibody titers at least five months post-infection, high enough to give hope for a protective and longer-lasting immunity after wild-type SARS-CoV-2 infection, which may also be relevant to estimate the long-term efficacy of a vaccination. Furthermore, our data reveal that individuals who have recovered from symptomatic COVID-19 generated more robust neutralizing antibody responses than those with asymptomatic infections.

\section{ACKNOWLEDGEMENTS}

We gratefully acknowledge the financial support of the Österreichische Forschungsförderungsgesellschaft (FFG), grant no. 35863961. The authors report no potential conflict of interest. We also thank DDI Martin Lamprecht, Stefanie Sonnleitner and Mag. Eva Hinterbichler for excellent technical assistance and valuable suggestions.

\section{ETHICAL STATEMENT}

The authors confirm that the ethical policies of the journal, as noted on the journal's author guidelines page, have been adhered to and the appropriate ethical review committee approval has been received (Ethics Committee of the University Hospital Wuerzburg no. 20201105_01).

\section{CONFLICT OF INTEREST}

The authors have no conflict of interest to declare.

\section{REFERENCES}

Table 1: Comparison of different serologic methods for antibody detection in 34 COVID-19 patients one respectively five months post infection.

\begin{tabular}{llllllllll}
\hline T1 & positive & {$[\%]$} & negative & {$[\%]$} & borderline & {$[\%]$} & n. a. & total \\
\hline ELNA & 30 & 90.9 & 2 & 5.0 & 1 & 3.0 & 1 & 34 \\
IFA & 32 & 94.1 & 2 & 4.9 & 0 & 0.0 & 0 & 34 \\
CLIA IgG & 28 & 82.4 & 5 & 7.9 & 1 & 2.9 & 0 & 34 \\
IFA IgM & 11 & 32.4 & 23 & 67.6 & 0 & 0 & 0 & 34 \\
Total & 34 & 100.0 & & 2.9 & & 0.0 & & \\
T5 & positive & {$[\%]$} & negative & {$[\%]$} & borderline & {$[\%]$} & n. a. & total \\
ELNA & 29 & 85.3 & 5 & 14.7 & 0 & 0.0 & 0 & 34 \\
IFA & 21 & 65.6 & 5 & 15.6 & 6 & 18.8 & 2 & 34 \\
CLIA IgG & 27 & 81.8 & 5 & 15.2 & 1 & 3.0 & 1 & 34 \\
IFA IgM & 0 & 0.0 & 34 & 100.0 & 0 & 0.0 & 0 & 34 \\
\hline
\end{tabular}

n.a.: not assessed. 
Table 2: Development of SARS-CoV-2 specific humoral immunity within the first five months (22 weeks) post infection.

\begin{tabular}{llllllll}
\hline & loss & {$[\%]$} & increase & {$[\%]$} & constant & {$[\%]$} & total (without negatives) \\
\hline NT & 1 & 3.2 & 2 & 6.5 & 28 & 90.3 & 31 \\
IFA IgG & 11 & 34.4 & 0 & 0.0 & 21 & 65.6 & 32 \\
CLIA IgG & 7 & 21.9 & 7 & 21.9 & 18 & 56.3 & 32 \\
IFA IgM & 11 & 100 & 0 & 0.0 & 0 & 0.0 & 11 \\
\hline
\end{tabular}

Only patients with borderline or positive results in the corresponding tests were used for calculation. In NT, one patient $(3.2 \%)$ lost antibodies, two $(6.5 \%)$ developed higher, more stable IgG titers, the antibody titers of 28 patients $(90.3 \%)$ stayed constant during the first five months post infection.

Figure 1: The median titer of $\mathbf{s}$ ymptomatic patients was two levels higher than those of asymptomatic patients.

Figure 2: Development of neutralizing antibodies goes along with disease severity. Neutralization activity is more pronounced in patients with more severe infections. Moderate and severe courses of disease led to neutralizing antibodies in $100 \%$ of the patients, whereas $33 \%$ and $20 \%$ of persons with an asymptomatic course of infection did not constantly develop neutralizing antibodies.

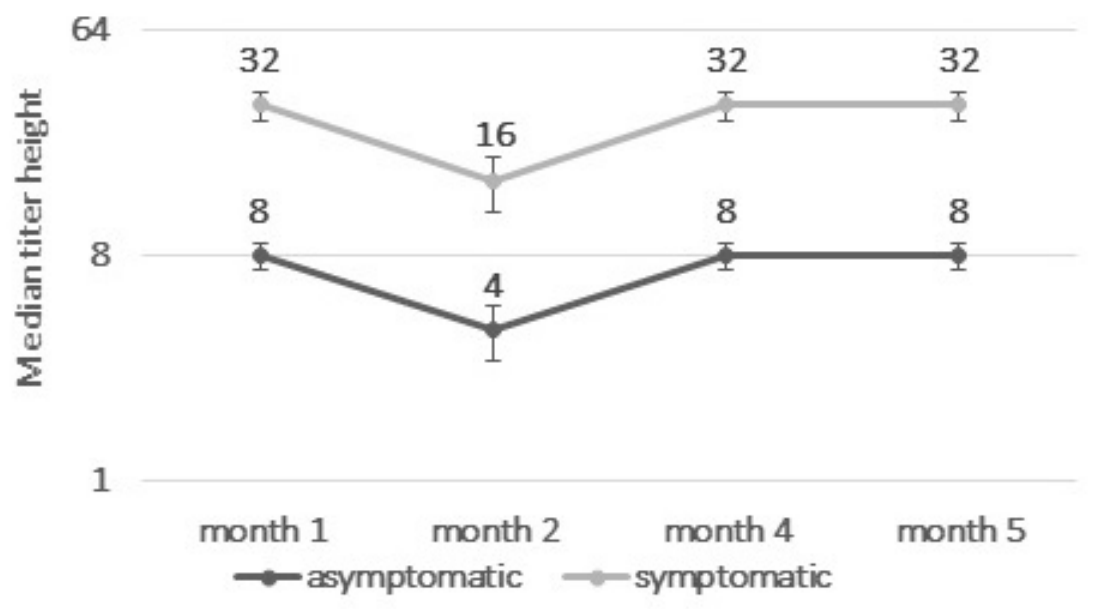




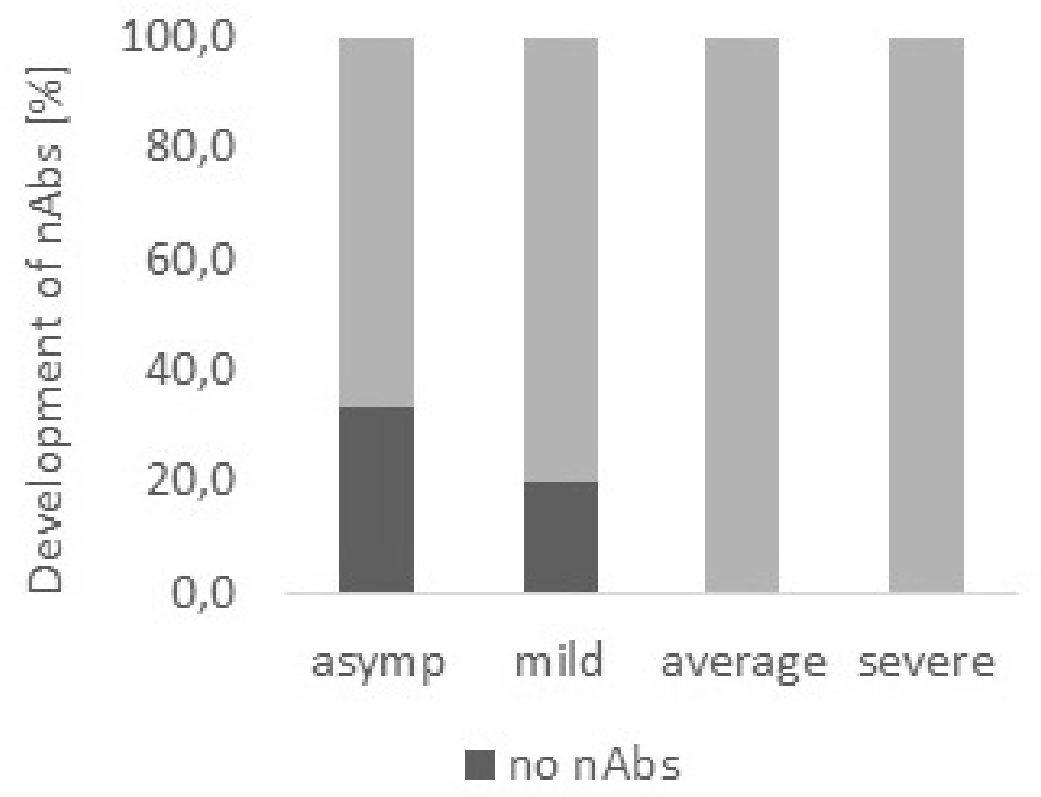

